\title{
A NOTE ON THE MARCHENKO-PASTUR LAW FOR A CLASS OF RANDOM MATRICES WITH DEPENDENT ENTRIES
}

\author{
SEAN O'ROURKE
}

\begin{abstract}
We consider a class of real random matrices with dependent entries and show that the limiting empirical spectral distribution is given by the Marchenko-Pastur law. Additionally, we establish a rate of convergence of the expected empirical spectral distribution.
\end{abstract}

\section{Introduction and Main Results}

Suppose $M_{n}$ is a $n \times n$ matrix with real eigenvalues $\lambda_{1}, \lambda_{2}, \ldots, \lambda_{n}$. Then the empirical spectral distribution (ESD) of the matrix $M_{n}$ is defined by

$$
F^{M_{n}}(x):=\frac{\#\left\{1 \leq i \leq n: \lambda_{i} \leq x\right\}}{n} .
$$

We will be interested in the case when $M_{n}:=\frac{1}{n} A_{n} A_{n}^{\mathrm{T}}$ and $A_{n}$ is an $n \times N$ real random matrix.

If the entries of $A_{n}$ are i.i.d. random variables with zero mean and variance one, we call $M_{n}$ a sample covariance matrix. There are many results concerning the limiting behavior of the spectral distribution of sample covariance matrices. For example, Marchenko and Pastur ([17) and Wachter (21]) prove that the ESD $F^{\frac{1}{n}} A_{n} A_{n}^{\mathrm{T}}(x)$ converges to $F_{c}(x)$ provided that $N / n \rightarrow c \in(0, \infty)$, where $F_{c}$ is the distribution function for the Marchenko-Pastur law with parameter $c>0$. That is, $F_{c}$ has density

$$
p_{c}(x)= \begin{cases}\frac{\sqrt{(x-a)(b-x)}}{2 \pi x} & : a \leq x \leq b, \\ 0 & : \text { otherwise }\end{cases}
$$

and a point mass $1-c$ at the origin if $c<1$, where $a=(1-\sqrt{c})^{2}$ and $b=(1+\sqrt{c})^{2}$. The above convergence holds with probability 1 (see for example [5] and $[$, Chapter 3]).

There are a number of results in which the independence assumption (on the entries of $A_{n}$ ) is weakened. In the seminal paper by Marchenko and Pastur [17, one considers independent rows rather than independent entries. In 22, Yin and Krishnaiah consider the case where the independent rows have a spherically symmetric distribution.

More recently in 2006, Aubrun obtained the Marchenko-Pastur law for matrices with independent rows distributed uniformly on the $l_{p}^{n}$ balls, 4]. This was generalized by Pajor and Pastur in [20] to matrices with independent rows distributed according to an arbitrary isotropic log-concave measure.

In [13] and [15, Götze and Tikhomirov study two classes of random matrices which generalize Wigner random matrices and sample covariance random matrices. In particular, these matrices satisfy certain martingale-type conditions without any 
assumption on the independence of the entries. In a similar setting, Adamczak studied a class of random matrices with uncorrelated entries in which each normalized row and normalized column converges to one in probability, [1].

Other random matrix ensembles with dependent entries that have been studied include random Markov matrices with independent rows and doubly stochastic random matrices (see [10, 9, 11] and references contained therein).

In this note, we study a class of random matrices with dependent entries and show that the limiting empirical distribution of the eigenvalues is given by the Marchenko-Pastur law. In particular, we consider a sequence of $n \times N$ random matrices $A_{n}$ with the following properties.

Definition 1 (Condition C0). Let $\left\{A_{n}\right\}_{n>1}$ be a sequence of $n \times N$ real random matrices where $N=N(n)$ and $c_{n}:=N / n$. We let $r_{1}^{(n)}, \ldots, r_{n}^{(n)}$ denote the rows of $A_{n}=\left(\zeta_{i j}^{(n)}\right)_{1 \leq i \leq n, 1 \leq j \leq N}$ and define the $\sigma$-algebra associated to row $k$ as

$$
\mathcal{F}_{k}^{(n)}:=\sigma\left(r_{1}^{(n)}, \ldots, r_{k-1}^{(n)}, r_{k+1}^{(n)}, \ldots, r_{n}^{(n)}\right)
$$

for all $k=1, \ldots, n$. Let $\mathbb{E}_{k}[\cdot]$ denote the conditional expectation with respect to the $\sigma$-algebra associated to row $k$. We then say that the sequence $\left\{A_{n}\right\}_{n \geq 1}$ obeys condition $\mathbf{C O}$ if the following hold:

(i) $\mathbb{E}_{k}\left[\zeta_{k i}^{(n)}\right]=0$ for all $i, k, n$

(ii) One has

$$
q_{n}:=\sup _{k} \frac{1}{n} \sum_{i=1}^{N} \mathbb{E}\left|\mathbb{E}_{k}\left[\left(\zeta_{k i}^{(n)}\right)^{2}\right]-1\right|=o(1)
$$

(iii) One has

$$
\sup _{k, i \neq j}\left|\mathbb{E}_{k}\left[\zeta_{k i}^{(n)} \zeta_{k j}^{(n)}\right]\right|+\sup _{k, i, j \neq l}\left|\mathbb{E}_{k}\left[\left(\zeta_{k i}^{(n)}\right)^{2} \zeta_{k j}^{(n)} \zeta_{k l}^{(n)}\right]\right|=O\left(n^{-1 / 2} \gamma_{n}\right)
$$

a.s., where $\gamma_{n} \rightarrow 0$ as $n \rightarrow \infty$.

(iv) $\sup \left|\mathbb{E}_{k}\left[\zeta_{k i}^{(n)} \zeta_{k j}^{(n)} \zeta_{k l}^{(n)} \zeta_{k m}^{(n)}\right]\right|=O\left(n^{-1} \gamma_{n}\right)$ a.s where the supremum is over all $k$ and all $i, j, l, m$ distinct.

(v) $\sup _{n, i, j} \mathbb{E}\left|\zeta_{i j}^{(n)}\right|^{4} \leq M<\infty$

(vi) One has

$$
\rho_{n}:=\sup _{k} \frac{1}{n^{2}} \sum_{1 \leq i, j \leq N} \mathbb{E}\left|\mathbb{E}_{k}\left[\left(\zeta_{k i}^{(n)}\right)^{2}\left(\zeta_{k j}^{(n)}\right)^{2}\right]-1\right|=o(1) .
$$

(vii) There exists a non-negative integer sequence $\beta_{n}=o(\sqrt{n})$ such that $\sigma\left(r_{i_{1}}^{(n)}, \ldots, r_{i_{k}}^{(n)}\right)$ and $\sigma\left(r_{j_{1}}^{(n)}, \ldots, r_{j_{m}}^{(n)}\right)$ are independent $\sigma$-algebras whenever

$$
\min _{1 \leq l \leq k, 1 \leq p \leq m}\left|i_{l}-j_{p}\right|>\beta_{n} .
$$

Remark 2. Condition (ii) implies that entries from different rows are uncorrelated while (iii) and (iv) allow for a weak correlation amongst entries in the same row. Condition (iii) is a requirement on the variance of the entries and (v) is a moment assumption on the entries. Condition (vi) is of a technical nature. In particular, 
(vii) (along with (iii) allows one to control terms of the form

$$
\sup _{k} \operatorname{Var}\left(\frac{1}{n}\left|r_{k}^{(n)}\right|^{2}\right)
$$

where $\left|r_{k}^{(n)}\right|$ is the Euclidian norm of the vector $r_{k}^{(n)}$. In words, condition (vii) implies that rows, which are "far enough apart," are independent.

Example 3. Let $\xi$ be a real random variable with mean zero, variance one, and $\mathbb{E}\left|\xi^{4}\right|<\infty$. Let $A_{n}$ be an $n \times N$ matrix where each entry is an i.i.d. copy of $\xi$. If $N / n \rightarrow c \in(0, \infty)$, then $A_{n}$ satisfies Definition 1 All the results in this paper are already known for such matrices with i.i.d. entries. See for example [17, [8, Chapter 3], [5], and references contained therein.

Example 4. Let $A_{n}$ be a $n \times(2 n)$ matrix where the rows are i.i.d. random vectors such that the entries of $r_{k}^{(n)}$ are \pm 1 symmetric Bernoulli random variables chosen uniformly such that the sum of the entries of each row is zero. Then the sequence $\left\{A_{n}\right\}_{n \geq 1}$ obeys condition C0. Indeed, one can compute

$$
\begin{aligned}
\mathbb{E}\left[\zeta_{i j}^{(n)}\right] & =0, \\
\operatorname{Var}\left[\zeta_{k i}^{(n)}\right] & =1, \\
\mathbb{E}\left[\zeta_{k i}^{(n)} \zeta_{k j}^{(n)}\right] & =-\frac{1}{2 N-1} \text { for } i \neq j,
\end{aligned}
$$

and

$$
\mathbb{E}\left[\zeta_{k i}^{(n)} \zeta_{k j}^{(n)} \zeta_{k l}^{(n)} \zeta_{k m}^{(n)}\right]=\frac{12 N^{2}-12 N}{2 N(2 N-1)(2 N-2)(2 N-3)}=O\left(\frac{1}{N^{2}}\right)
$$

for $i, j, l, m$ distinct, where $N=2 n$. In particular, one finds that $\gamma_{n}=n^{-1 / 2}$ and $q_{n}, \rho_{n}, \beta_{n}=0$.

Let us mention that the conditions in Definition 1 1 are similar to the assumptions of Theorem 1 in [17. However, in [17, the authors require the rows of $A_{n}$ to be independent.

Also, the sequence of random matrices defined in Example 4 satisfies condition C0, but does not satisfy the assumptions of the theorems provided in [4, 20], 13], or 1 .

Let $\|M\|$ denote the spectral norm of the matrix $M$. In this paper, we shall prove the following theorems.

Theorem 5. Let $\left\{A_{n}\right\}_{n \geq 1}$ be a sequence of real random matrices that obey condition $\mathbf{C 0}$ and assume $c_{n}:=N / n \rightarrow c \in(0, \infty)$. Then

$$
\left\|\mathbb{E} F^{\frac{1}{n} A_{n} A_{n}^{\mathrm{T}}}-F_{c}\right\|:=\sup _{x}\left|\mathbb{E} F^{\frac{1}{n} A_{n} A_{n}^{\mathrm{T}}}-F_{c}\right| \longrightarrow 0
$$

as $n \rightarrow \infty$. Moreover, if there exists $p>1$ such that

$$
\sum_{n=1}^{\infty} \frac{\left(\beta_{n}+1\right)^{p}}{n^{p / 2}}<\infty
$$

then

$$
\left\|F^{\frac{1}{n} A_{n} A_{n}^{\mathrm{T}}}-F_{c}\right\| \longrightarrow 0
$$

almost surely as $n \rightarrow \infty$. 
Theorem 6. Let $\left\{A_{n}\right\}_{n \geq 1}$ be a sequence of real random matrices that obey condition $\mathbf{C 0}$ and assume $c_{n}:=N / n \geq 1$ such that $c_{n} \rightarrow c \in[1, \infty)$. Additionally assume that

$$
\limsup _{n \rightarrow \infty} \frac{1}{n} \mathbb{E}\left\|A_{n} A_{n}^{\mathrm{T}}\right\|<\infty
$$

Then we obtain that

$$
\left\|\mathbb{E} F^{\frac{1}{n} A_{n} A_{n}^{\mathrm{T}}}-F_{c_{n}}\right\|=O\left(\max \left(q_{n}^{1 / 22}, \gamma_{n}^{1 / 22}, \rho_{n}^{1 / 22},\left(\frac{\left(\beta_{n}+1\right)^{2}}{n}\right)^{1 / 22}\right)\right) .
$$

Remark 7. We stated Theorem 5 for a sequence of random matrices that obey condition C0. However, it is actually possible to prove the convergence of the expected ESD without condition (vii) from Definition 11 That is, if the sequence $\left\{A_{n}\right\}_{n \geq 1}$ satisfies conditions (ii) - (vi) from Definition 1 with $c_{n} \rightarrow c \in(0, \infty)$, then

$$
\left\|\mathbb{E} F^{\frac{1}{n} A_{n} A_{n}^{\mathrm{T}}}-F_{c}\right\| \longrightarrow 0
$$

as $n \rightarrow \infty$. The proof of this statement repeats the proof of Theorem 5 almost exactly. We detail the necessary changes in Remark 14. It should be noted that the almost sure convergence portion of Theorem 5still requires condition (vii) from Definition 1 and (1).

Remark 8. Without any additional information on the convergence rate of $c_{n}$ to $c$, we cannot obtain a rate of convergence of $\left\|\mathbb{E} F^{A_{n} A_{n}^{\mathrm{T}}}-F_{c}\right\|$. This is why $F_{c_{n}}$ appears in Theorem 6 .

Remark 9. The rates obtained in Theorem 6 are not optimal and are obtained as a simple corollary to Lemma 13 below.

Example 10. Let $\left\{A_{n}\right\}_{n \geq 1}$ be the sequence of random matrices defined in Example 4. Theorem 5 implies that

$$
\left\|F^{\frac{1}{n} A_{n} A_{n}^{\mathrm{T}}}-F_{2}\right\| \longrightarrow 0
$$

almost surely as $n \rightarrow \infty$. We will now use Theorem 6 to obtain a rate of convergence for $\mathbb{E} F^{\frac{1}{n}} A_{n} A_{n}^{\mathrm{T}}$. We must verify that (2) holds. By [2, Theorem 3.13] 1], there exists $C, C^{\prime}>0$ such that for any $0<\epsilon<1 / 3$,

$$
\mathbb{P}\left(\left\|A_{n} A_{n}^{\mathrm{T}}\right\| \geq C n\right) \leq C^{\prime} \frac{\log n}{n^{1+\epsilon}} .
$$

Since we always have the bound

$$
\left\|A_{n} A_{n}^{\mathrm{T}}\right\| \leq \operatorname{Tr}\left(A_{n} A_{n}^{\mathrm{T}}\right)=n^{2},
$$

it follows that

$$
\mathbb{E}\left\|A_{n} A_{n}^{\mathrm{T}}\right\|=O(n)
$$

\footnotetext{
${ }^{1}$ One technical assumption required by Theorem 3.13 is control over the $\psi_{1}$-norm $\left(\|\cdot\|_{\psi_{1}}\right)$ of the term $|\langle\xi, y\rangle|$ where $\xi$ is a row of the matrix $A_{n}$ and $y$ is an arbitrary unit vector. In particular, one can show that$$
\|\langle\xi, y\rangle\|_{\psi_{1}} \leq \frac{\sqrt{n}}{\log n^{1+\epsilon}} .
$$

The bound follows by applying Markov's inequality, which yields

$$
\mathbb{P}(|\langle\xi, y\rangle|>t)=O\left(t^{-4}\right),
$$

and then taking $t=n^{1 / 3}$.
} 
Therefore, Theorem 6 gives the rate of convergence

$$
\left\|\mathbb{E} F^{\frac{1}{n} A_{n} A_{n}^{\mathrm{T}}}-F_{2}\right\|=O\left(n^{-1 / 44}\right) .
$$

\section{Stieltues Transform}

If $G(x)$ is a function of bounded variation on the real line, then its Stieltjes transform is defined by

$$
S_{G}(z)=\int \frac{1}{x-z} d G(x)
$$

for $z \in D:=\{z \in \mathbb{C}: \operatorname{Im}(z)>0\}$.

Let $m_{c}(z)$ be the Stieltjes transform of $F_{c}$, the distribution function of the Marchenko-Pastur law with parameter $c$. One can then check (see for example [8]), that

$$
m_{c}(z)=\frac{-(z+1-c)+\sqrt{(z+1-c)^{2}-4 z}}{2 z} .
$$

Furthermore, $m_{c}(z)$ can be characterized uniquely as the solution to

$$
m_{c}(z)=\frac{1}{c-1-z-z m_{c}(z)}
$$

that satisfies $\operatorname{Im}\left(z m_{c}(z)\right) \geq 0$ for all $z$ with $\operatorname{Im} z>0$.

We will study the Stieltjes transform of the ESD of the random matrix $\frac{1}{n} A_{n} A_{n}^{\mathrm{T}}$ in order to prove Theorems 5 and 6 . In particular, the following lemma states that it suffices to show the convergence of the Stieltjes transform of the ESD to the Stieltjes transform of $F_{c}$.

Lemma 11 ([8, Theorem B.9]). Assume that $\left\{G_{n}\right\}$ is a sequence of functions of bounded variation and $G_{n}(-\infty)=0$ for all $n$. Then

$$
\lim _{n \rightarrow \infty} s_{G_{n}}(z)=s(z) \quad \forall z \in D
$$

if and only if there is a function of bounded variation $G$ with $G(-\infty)=0$ and Stieltjes transform $s(z)$ and such that $G_{n} \rightarrow G$ vaguely.

We will also use the following lemma in order to establish the rate of convergence in Theorem 6.

Lemma 12 ( [8, Theorem B.14] ). Let $F$ be a distribution function and let $G$ be a function of bounded variation satisfying $\int|F(x)-G(x)| d x<\infty$. Denote their Stieltjes transforms by $s_{F}(z)$ and $s_{G}(z)$ respectively, where $z=u+i v \in D$. Then

$$
\begin{aligned}
\|F-G\| \leq & \frac{1}{\pi(1-\xi)(2 \rho-1)}\left(\int_{-A}^{A}\left|s_{F}(z)-s_{G}(z)\right| d u\right. \\
& +2 \pi v^{-1} \int_{|x|>B}|F(x)-G(x)| d x \\
& \left.+v^{-1} \sup _{x} \int_{|u| \leq 2 v a}|G(x+u)-G(x)| d u\right),
\end{aligned}
$$

where the constants $A>B>0, \xi$, and a are restricted by $\rho=\frac{1}{\pi} \int_{|u| \leq a} \frac{1}{u^{2}+1} d u>\frac{1}{2}$, and $\xi=\frac{4 B}{\pi(A-B)(2 \rho-1)} \in(0,1)$. 


\section{Proof of Theorems 5 and 6}

Let $\left\{A_{n}\right\}_{n \geq 1}$ be a sequence of real random matrices that obeys condition C0 and assume $c_{n}:=N / n \rightarrow c \in(0, \infty)$. We begin by introducing some notation. Let

$$
s_{n}(z):=\frac{1}{n} \operatorname{Tr}\left(\frac{1}{n} A_{n} A_{n}^{\mathrm{T}}-z I_{n}\right)^{-1}=\frac{1}{n} \operatorname{Tr}\left(\frac{1}{n} A_{n} A_{n}^{\mathrm{T}}-z\right)^{-1}
$$

where $I_{n}$ is the identity matrix of order $n$ and $z=u+i v$. Fix $\alpha>0$ and let

$$
D_{\alpha, n}:=\left\{z=u+i v \in \mathbb{C}:|u| \leq \alpha, v_{n} \leq v \leq 1\right\}
$$

where $v_{n}$ is a sequence we will choose later such that $0<v_{n}<1$ for all $n$. We will eventually allow the sequence $v_{n}$ to approach zero as $n$ tends to infinity.

We will use the following lemma to prove Theorems 5 and 6 .

Lemma 13. Suppose $\left\{A_{n}\right\}_{n \geq 1}$ is a sequence of real random matrices that obey condition $\boldsymbol{C O}$ and assume $c_{n}:=N / n \rightarrow c \in(0, \infty)$. Then for any $\alpha>0$

$$
\sup _{z \in D_{\alpha, n}}\left|\mathbb{E} s_{n}(z)-\frac{1}{c_{n}-1-z-z \mathbb{E} s_{n}(z)}\right|=O\left(\frac{\sqrt{q_{n}}+\sqrt{\gamma_{n}}+\sqrt{\rho_{n}}}{v_{n}^{3}}+\frac{\beta_{n}+1}{\sqrt{n} v_{n}^{3}}\right) .
$$

We prove Lemma 13 in Section 4. For the moment, assume this lemma. First, take $v_{n}>0$ to be fixed. Then $D_{\alpha, n}$ does not change with $n$. Since $c_{n} \rightarrow c$, we obtain that

$$
\mathbb{E} s_{n}(z)=\frac{1}{c-1-z-z \mathbb{E} s_{n}(z)}+o(1)
$$

for all $z \in D_{\alpha, n}$. Fix $z_{0}=u_{0}+i v_{0} \in D_{\alpha, n}$. Since $\left|\mathbb{E} s_{n}\left(z_{0}\right)\right| \leq \frac{1}{v_{0}}$, one can use a compactness argument to obtain a convergent subsequence $\mathbb{E} s_{n_{k}}\left(z_{0}\right) \rightarrow s\left(z_{0}\right)$. Then $s\left(z_{0}\right)$ must satisfy the equation

$$
s\left(z_{0}\right)=\frac{1}{c-1-z_{0}-z_{0} s\left(z_{0}\right)} .
$$

Also, since the eigenvalues of $A_{n} A_{n}^{\mathrm{T}}$ are non-negative, $\operatorname{Im}\left(z \mathbb{E} s_{n}(z)\right) \geq 0$ for all $\operatorname{Im}(z)>0$ and hence $\operatorname{Im}\left(z_{0} s\left(z_{0}\right)\right) \geq 0$. Thus, by the characterization (3), it follows that $s\left(z_{0}\right)=m_{c}\left(z_{0}\right)$. Since every convergent subsequence of $\left\{\mathbb{E} s_{n}\left(z_{0}\right)\right\}$ must converge to the same limit, we obtain that

$$
\lim _{n \rightarrow \infty} \mathbb{E} s_{n}\left(z_{0}\right)=m_{c}\left(z_{0}\right)
$$

and since $z_{0} \in D_{\alpha, n}$ was arbitrary, one obtains

$$
\lim _{n \rightarrow \infty} \mathbb{E} s_{n}(z)=m_{c}(z)
$$

for all $z \in D_{\alpha, n}$. Finally, since $\left|\mathbb{E} s_{n}(z)\right| \leq \frac{1}{v}$, Vitali's Convergence Theorem implies that (44) holds for all $z \in D$. Therefore,

$$
\left\|\mathbb{E} F^{\frac{1}{n} A_{n} A_{n}^{\mathrm{T}}}-F_{c}\right\|:=\sup _{x}\left|\mathbb{E} F^{\frac{1}{n} A_{n} A_{n}^{\mathrm{T}}}-F_{c}\right| \longrightarrow 0
$$

as $n \rightarrow \infty$.

To obtain the almost sure convergence in Theorem 5, one repeats the argument above and then applies the Borel-Cantelli lemma, since

$$
\mathbb{P}\left(\left|s_{n}(z)-\mathbb{E} s_{n}(z)\right| \geq \epsilon\right) \leq \frac{C_{p}\left(\beta_{n}+1\right)^{p}}{n^{p / 2} v_{n}^{p} \epsilon^{p}}
$$

by Lemma 15 from Appendix $\mathrm{A}$ 
To prove Theorem 6, we will apply Lemma 12, Under assumption (2), there exists $B>0$ such that

$$
\mathbb{E} F^{\frac{1}{n} A_{n} A_{n}^{\mathrm{T}}}(x)-F_{c_{n}}(x)=0
$$

for all $|x|>B$ and $n$ sufficiently large.

By [8, Lemma 8.15], it follows that

$$
\sup _{x} \int_{|u|<v_{n}}\left|F_{c_{n}}(x+u)-F_{c_{n}}(x)\right|=O\left(v_{n}^{3 / 2}\right)
$$

for $c_{n} \geq 1$.

From Lemma 13, we have that

$$
\mathbb{E} s_{n}(z)=\frac{1}{c_{n}-1-z-z \mathbb{E} s_{n}(z)}+\delta_{n}
$$

for all $z \in D_{\alpha, n}$. Thus

$$
z\left(\mathbb{E} s_{n}(z)\right)^{2}+\mathbb{E} s_{n}(z)\left(z+1-c_{n}\right)+1=\delta_{n}\left(1-c_{n}-z-z \mathbb{E} s_{n}(z)\right) .
$$

By subtracting the quadratic equation for $m_{c_{n}}(z)$ obtained from (3), one finds that

$$
\left|\mathbb{E} s_{n}(z)-m_{c_{n}}(z)\right|=\frac{\left|\delta_{n}\right|\left|1-c_{n}+z+z \mathbb{E} s_{n}(z)\right|}{\left|z \mathbb{E} s_{n}(z)+z m_{c_{n}}(z)+z+1-c_{n}\right|}=O\left(\frac{\delta_{n}}{v_{n}^{2}}\right) .
$$

Therefore, from Lemma 12, one obtains that

$$
\left\|\mathbb{E} F^{\frac{1}{n} A_{n} A_{n}^{\mathrm{T}}}-F_{c_{n}}\right\|=O\left(\frac{\sqrt{q_{n}}+\sqrt{\gamma_{n}}+\sqrt{\rho_{n}}}{v_{n}^{5}}+\frac{\beta_{n}+1}{\sqrt{n} v_{n}^{5}}+\sqrt{v_{n}}\right)
$$

and hence we can take

$$
v_{n}=\max \left(q_{n}^{1 / 11}, \gamma_{n}^{1 / 11}, \rho_{n}^{1 / 11},\left(\frac{\left(\beta_{n}+1\right)^{2}}{n}\right)^{1 / 11}\right) .
$$

The proof of Theorem 6 is complete.

It only remains to prove Lemma 13

\section{Proof of Lemma 13}

Let $\left\{A_{n}\right\}_{n \geq 1}$ be a sequence of real random matrices that obey condition $\mathbf{C 0}$ and assume $c_{n}:=N / n \rightarrow c \in(0, \infty)$. Fix $\alpha>0$. In order to simplify notation, we drop the superscript $(n)$ and write $\zeta_{i j}$ and $r_{k}$ for the entries of $A_{n}$ and the rows of $A_{n}$ respectively. We define the resolvent

$$
R_{n}(z):=\left(\frac{1}{n} A_{n} A_{n}^{\mathrm{T}}-z\right)^{-1} .
$$

Using the Schur complement, we obtain that

$$
\left(R_{n}(z)\right)_{k k}=\frac{1}{\frac{1}{n}\left|r_{k}\right|^{2}-z-\frac{1}{n} r_{k} A_{n, k}^{\mathrm{T}} R_{n, k}(z) A_{n, k} r_{k}^{\mathrm{T}}}=: \frac{1}{a_{k}}
$$

where $A_{n, k}$ is obtained from the matrix $A_{n}$ by removing the $k$-th row and

$$
R_{n, k}=\left(\frac{1}{n} A_{n, k} A_{n, k}^{\mathrm{T}}-z\right)^{-1}
$$


Since $\left|\left(R_{n}(z)\right)_{k k}\right| \leq\left\|R_{n}(z)\right\| \leq \frac{1}{v_{n}}$, we obtain that $\left|a_{k}\right| \geq v_{n}$. Thus,

$$
\left|\mathbb{E} s_{n}(z)-\frac{1}{n} \sum_{k=1}^{n} \frac{1}{\mathbb{E} a_{k}}\right| \leq \frac{1}{n v_{n}^{2}} \sum_{k=1}^{n} \mathbb{E}\left|a_{k}-\mathbb{E} a_{k}\right| .
$$

We now compute the expectation of $a_{k}$. By condition (iii) in Definition 1, we have that

For convenience, write

$$
\left.\sup _{k}\left|\mathbb{E} \frac{1}{n}\right| r_{k}\right|^{2}-c_{n} \mid \leq q_{n}
$$

$$
B_{n, k}:=A_{n, k}^{\mathrm{T}} R_{n, k}(z) A_{n, k} .
$$

We first note that $\sup _{k}\left\|B_{n, k}\right\|=O\left(v_{n}^{-1}\right)$. Indeed, since $|z|^{2} \leq \alpha^{2}+1=O(1)$,

$$
\left\|B_{n, k}\right\|=\left\|R_{n, k}(z) A_{n, k} A_{n, k}^{\mathrm{T}}\right\|=\left\|I_{n-1}+z R_{n, k}(z)\right\| \leq 1+\frac{|z|}{v_{n}}
$$

for all $k=1, \ldots, n$. Then we have that

$$
\mathbb{E} \frac{1}{n} r_{k} B_{n, k} r_{k}^{\mathrm{T}}=\frac{1}{n} \sum_{i, j=1}^{N} \mathbb{E}\left[\mathbb{E}_{k}\left[\zeta_{k i} \zeta_{k j}\right]\left(B_{n, k}\right)_{i j}\right]=\frac{1}{n} \mathbb{E} \operatorname{Tr} B_{n, k}+\epsilon_{n, k}+O\left(\frac{q_{n}}{v_{n}}\right)
$$

uniformly for all $k$ (by condition (iii) in Definition 11) where

$$
\epsilon_{n, k}:=\frac{1}{n} \sum_{i \neq j} \mathbb{E}\left[\mathbb{E}_{k}\left[\zeta_{k i} \zeta_{k j}\right]\left(B_{n, k}\right)_{i j}\right] .
$$

By condition (iii), we have that

$$
\begin{aligned}
\left|\epsilon_{n, k}\right| & \leq\left(\mathbb{E} \frac{\gamma_{n}^{2}}{n^{3}} \sum_{i, j, s, t=1}^{N}\left|\left(B_{n, k}\right)_{i j}\right|\left|\left(B_{n, k}\right)_{s, t}\right|\right)^{1 / 2} \\
& \leq\left(\mathbb{E} \frac{2 \gamma_{n}^{2}}{n} \operatorname{Tr}\left(B_{n, k} B_{n, k}^{*}\right)\right)^{1 / 2}=O\left(v_{n}^{-1} \gamma_{n}\right)
\end{aligned}
$$

uniformly in $k$.

Combining the above yields,

$$
\sup _{k}\left|\mathbb{E} a_{k}-\left(c_{n}-z-\mathbb{E} \frac{1}{n} \operatorname{Tr} B_{n, k}\right)\right|=O\left(\frac{q_{n}+\gamma_{n}}{v_{n}}\right)
$$

We now note that $\frac{1}{n} \operatorname{Tr} B_{n, k}=\frac{n-1}{n}+z \frac{1}{n} \operatorname{Tr} R_{n, k}(z)$. By equation (3.11) in [7] (or alternatively, by Cauchy's Interlacing Theorem), one finds that

$$
\left|\frac{1}{n} \operatorname{Tr} R_{n, k}(z)-\frac{1}{n} \operatorname{Tr} R_{n}(z)\right|=O\left(\frac{1}{n v_{n}}\right)
$$

uniformly in $k$. Therefore, from (7) and the fact that $\frac{1}{n} \operatorname{Tr} R_{n}(z)=s_{n}(z)$, we obtain that

$$
\sup _{k}\left|\mathbb{E} a_{k}-\left(c_{n}-1-z-z \mathbb{E} s_{n}(z)\right)\right|=O\left(\frac{q_{n}+\gamma_{n}}{v_{n}}+\frac{1}{n v_{n}}\right)
$$

We now turn our attention to obtaining a bound for $\mathbb{E}\left|a_{k}-\mathbb{E} a_{k}\right|$. First we note that

$$
\left.\mathbb{E}\left|\frac{1}{n}\right| r_{k}\right|^{2}-c_{n} \mid \leq q_{n}
$$


by condition (iii) of Definition 1) Using (자) and the bounds obtained above for $\epsilon_{n, k}$, we have that

$$
\begin{aligned}
\mathbb{E}\left|\frac{1}{n} r_{k} B_{n, k} r_{k}^{\mathrm{T}}-\mathbb{E} \frac{1}{n} \operatorname{Tr} B_{n, k}\right|^{2}= & \frac{1}{n^{2}} \sum_{i, j, s, t=1}^{N} \mathbb{E}\left[\zeta_{k i} \zeta_{k j} \zeta_{k s} \zeta_{k t}\left(B_{n, k}\right)_{i j}\left(B_{n, k}\right)_{s t}\right] \\
& -\left(\mathbb{E} \frac{1}{n} \operatorname{Tr} B_{n, k}\right)^{2}+O\left(\frac{q_{n}+\gamma_{n}}{v_{n}^{2}}\right) .
\end{aligned}
$$

For the sum

$$
\frac{1}{n^{2}} \sum_{i, j, s, t=1}^{N} \mathbb{E}\left[\zeta_{k i} \zeta_{k j} \zeta_{k s} \zeta_{k t}\left(B_{n, k}\right)_{i j}\left(B_{n, k}\right)_{s t}\right]
$$

we consider several cases:

(a) When we sum over all $i, j, s, t$ distinct, one finds

$$
\frac{1}{n^{2}} \sum \mathbb{E}\left[\zeta_{k i} \zeta_{k j} \zeta_{k s} \zeta_{k t}\left(B_{n, k}\right)_{i j}\left(B_{n, k}\right)_{s t}\right]=O\left(\frac{\gamma_{n}}{v_{n}^{2}}\right)
$$

by condition (iv).

(b) When we sum of all $i=j, s=t$, we obtain

$$
\frac{1}{n^{2}} \sum \mathbb{E}\left[\zeta_{k i} \zeta_{k j} \zeta_{k s} \zeta_{k t}\left(B_{n, k}\right)_{i j}\left(B_{n, k}\right)_{s t}\right]=\mathbb{E}\left[\left(\frac{1}{n} \operatorname{Tr} B_{n, k}\right)^{2}\right]+O\left(\frac{\rho_{n}}{v_{n}^{2}}\right)
$$

by condition vil).

(c) When we sum over all $i=s, j=t$ ( or $i=t, j=s$ ), we have

$$
\begin{aligned}
\frac{1}{n^{2}} \sum \mathbb{E} & {\left[\zeta_{k i} \zeta_{k j} \zeta_{k s} \zeta_{k t}\left(B_{n, k}\right)_{i j}\left(B_{n, k}\right)_{s t}\right] } \\
= & \mathbb{E}\left[\frac{1}{n^{2}} \operatorname{Tr}\left(B_{n, k} B_{n, k}^{*}\right)\right]+O\left(\frac{\rho_{n}}{v_{n}^{2}}\right) \\
& =O\left(\frac{\rho_{n}}{v_{n}^{2}}+\frac{1}{n v_{n}^{2}}\right)
\end{aligned}
$$

by condition (vil).

(d) When $i=s, j \neq t$ (or $i=t, j \neq s$ ), one finds that

$$
\frac{1}{n^{2}} \sum \mathbb{E}\left[\zeta_{k i} \zeta_{k j} \zeta_{k s} \zeta_{k t}\left(B_{n, k}\right)_{i j}\left(B_{n, k}\right)_{s t}\right]=O\left(\frac{\gamma_{n}}{\sqrt{n} v_{n}^{2}}\right)
$$

by condition (iii).

(e) When $i=j, s \neq t$, we find

$$
\begin{aligned}
\frac{1}{n^{2}} \sum \mathbb{E} & {\left[\zeta_{k i} \zeta_{k j} \zeta_{k s} \zeta_{k t}\left(B_{n, k}\right)_{i j}\left(B_{n, k}\right)_{s t}\right] } \\
\leq & \frac{\gamma_{n}}{n^{3 / 2} v_{n}} \mathbb{E}\left(\sum_{s, t}\left|\left(B_{n, k}\right)_{s t}\right|\right) \\
\leq & \frac{\gamma_{n}}{n^{3 / 2} v_{n}} \mathbb{E}\left(\sum_{s, t, l, m}\left|\left(B_{n, k}\right)_{s t}\right|\left|\left(B_{n, k}\right)_{l m}\right|\right)^{1 / 2} \\
\leq & \frac{\gamma_{n}}{n^{3 / 2} v_{n}} n \mathbb{E}\left(2 \operatorname{Tr}\left(B_{n, k} B_{n, k}^{*}\right)\right)^{1 / 2}=O\left(\frac{\gamma_{n}}{v_{n}^{2}}\right)
\end{aligned}
$$


by Cauchy-Schwarz and condition (iii).

Therefore, we obtain that

$$
\mathbb{E}\left|\frac{1}{n} r_{k} B_{n, k} r_{k}^{\mathrm{T}}-\mathbb{E} \frac{1}{n} \operatorname{Tr} B_{n, k}\right|^{2}=\operatorname{Var}\left(\frac{1}{n} \operatorname{Tr} B_{n, k}\right)+O\left(\frac{q_{n}+\gamma_{n}+\rho_{n}}{v_{n}^{2}}+\frac{1}{n v_{n}^{2}}\right) .
$$

The bound in (10) holds uniformly in $k$ since the bounds in conditions (iii), (iv), and (vi) of Definition 1 hold uniformly in $k$.

By Lemma 15] in Appendix A, we have that

$$
\sup _{k} \operatorname{Var}\left(\frac{1}{n} \operatorname{Tr} B_{n, k}\right)=O\left(\frac{\left(\beta_{n}+1\right)^{2}}{n v_{n}^{2}}\right)
$$

and hence

$$
\mathbb{E}\left|\frac{1}{n} r_{k} B_{n, k} r_{k}^{\mathrm{T}}-\mathbb{E} \frac{1}{n} \operatorname{Tr} B_{n, k}\right|^{2}=O\left(\frac{q_{n}+\gamma_{n}+\rho_{n}}{v_{n}^{2}}+\frac{\left(\beta_{n}+1\right)^{2}}{n v_{n}^{2}}\right) .
$$

Therefore

$$
\sup _{k} \mathbb{E}\left|a_{k}-\mathbb{E} a_{k}\right|=O\left(\frac{\sqrt{q_{n}}+\sqrt{\gamma_{n}}+\sqrt{\rho_{n}}}{v_{n}}+\frac{\beta_{n}+1}{\sqrt{n} v_{n}}\right) .
$$

One can also observe that $\operatorname{Im}\left(z s_{n}(z)\right) \geq 0$ for all $z$ with $\operatorname{Im} z>0$, since the eigenvalues of $A_{n} A_{n}^{\mathrm{T}}$ are non-negative. Combining this fact with equations (5) and (7) and the estimates above, we obtain that

$$
\left|\mathbb{E} s_{n}(z)-\frac{1}{1-c_{n}-z-z s_{n}(z)}\right|=O\left(\frac{\sqrt{q_{n}}+\sqrt{\gamma_{n}}+\sqrt{\rho_{n}}}{v_{n}^{3}}+\frac{\beta_{n}+1}{\sqrt{n} v_{n}^{3}}\right)
$$

where the bound holds uniformly for $z \in D_{\alpha, n}$. The proof of Lemma[13 is complete.

Remark 14. As noted in Remark 7, it is possible to show that if the sequence $\left\{A_{n}\right\}_{n \geq 1}$ satisfies conditions (ii) - (vi) from Definition 1 with $c_{n} \rightarrow c \in(0, \infty)$, then

$$
\left\|\mathbb{E} F^{\frac{1}{n} A_{n} A_{n}^{\mathrm{T}}}-F_{c}\right\| \longrightarrow 0
$$

as $n \rightarrow \infty$. The proof of the above statement repeats the proof of Theorem 5 almost exactly; we now detail the necessary changes.

Since the Stieltjes transform is an analytic and bounded function, it suffices to prove the convergence of $\mathbb{E} s_{n}(z)$ to $m_{c}(z)$ for all $z$ in a compact set in the upper-half plane with $\operatorname{Im}(z) \geq \kappa$ for a sufficiently large constant $\kappa$ to be chosen later.

A careful reading of the proof of Lemma 13 reveals that condition (vii) from Definition 1 is only used to invoke Lemma 15 and obtain the variance bound (16). Thus, in order to prove (13), it suffices to show that

$$
\operatorname{Var}\left(\frac{1}{n} \operatorname{Tr} R_{n}(z)\right)=o(1)
$$

for all $z$ in a compact set in the upper-half plane with $\operatorname{Im}(z) \geq \kappa$.

We decompose

$$
\begin{aligned}
\left(R_{n}(z)\right)_{k k} & =\frac{1}{\frac{1}{n}\left|r_{k}\right|^{2}-z-\frac{1}{n} r_{k} A_{n, k}^{\mathrm{T}} R_{n, k}(z) A_{n, k} r_{k}^{\mathrm{T}}} \\
& =\frac{1}{c_{n}-1-z-z \mathbb{E} s_{n}(z)-\epsilon_{k}}
\end{aligned}
$$


where

$$
\epsilon_{k}=c_{n}-1-z \mathbb{E} s_{n}(z)-\frac{1}{n}\left|r_{k}\right|^{2}+\frac{1}{n} r_{k} A_{n, k}^{\mathrm{T}} R_{n, k}(z) A_{n, k} r_{k}^{\mathrm{T}} .
$$

Thus

$$
\begin{aligned}
\left(R_{n}(z)\right)_{k k}= & \frac{1}{c_{n}-1-z-z \mathbb{E} s_{n}(z)} \\
& +\frac{\epsilon_{k}}{\left(c_{n}-1-z-z \mathbb{E} s_{n}(z)\right)\left(\left(c_{n}-1-z-z \mathbb{E} s_{n}(z)-\epsilon_{k}\right)\right.} \\
= & \frac{1}{c_{n}-1-z-z \mathbb{E} s_{n}(z)}\left[1+\left(R_{n}(z)\right)_{k k} \epsilon_{k}\right] .
\end{aligned}
$$

Taking $\operatorname{Im}(z) \geq \kappa$ we obtain

$$
\operatorname{Var}\left(\frac{1}{n} \operatorname{Tr} R_{n}(z)\right) \leq \frac{C}{\kappa^{4} n} \sum_{k=1}^{n} \mathbb{E}\left|\epsilon_{k}\right|^{2}
$$

for some absolute constant $C>0$. Using condition (vi) from Definition 10 (8), and (10), we bound $\mathbb{E}\left|\epsilon_{k}\right|^{2}$ and obtain

$$
\operatorname{Var}\left(\frac{1}{n} \operatorname{Tr} R_{n}(z)\right) \leq \frac{C|z|^{2}}{\kappa^{4}} \operatorname{Var}\left(\frac{1}{n} \operatorname{Tr} R_{n}(z)\right)+O\left(q_{n}+\gamma_{n}+\rho_{n}+\frac{1}{\sqrt{n}}\right) .
$$

Taking $z$ in a compact set for which $|z|^{2} / \kappa^{4}$ is sufficiently small verifies (14).

\section{Appendix A. Estimate of Variance of Stieltues Transform}

Lemma 15. Let $A_{n}$ be an $n \times N$ real random matrix with rows $r_{1}^{(n)}, \ldots, r_{n}^{(n)}$ that satisfy condition (vii) from Definition 1. Then for every $p>1$ there exists a constant $C_{p}>0$ (depending only on $p$ ) such that

$$
\mathbb{E}\left|\frac{1}{n} \operatorname{Tr}\left(\frac{1}{n} A_{n} A_{n}^{\mathrm{T}}-z I_{n}\right)^{-1}-\frac{1}{n} \mathbb{E} \operatorname{Tr}\left(\frac{1}{n} A_{n} A_{n}^{\mathrm{T}}-z I_{n}\right)^{-1}\right|^{p} \leq \frac{C_{p}\left(\beta_{n}+1\right)^{p}}{n^{p / 2}|\operatorname{Im} z|^{p}}
$$

for any $z$ with $\operatorname{Im} z \neq 0$. In particular, there exists an absolute constant $C>0$ such that

$$
\operatorname{Var}\left[\frac{1}{n} \operatorname{Tr}\left(\frac{1}{n} A_{n} A_{n}^{\mathrm{T}}-z I_{n}\right)^{-1}\right] \leq \frac{C\left(\beta_{n}+1\right)^{2}}{n|\operatorname{Im} z|^{2}}
$$

for any $z$ with $\operatorname{Im} z \neq 0$.

Proof. Since (15) implies (16) when $p=2$, it suffices to prove (15) for arbitrary $p>1$. Let $\mathbb{E}_{\leq k}$ denote the conditional expectation with respect to the $\sigma$-algebra generated by $r_{1}^{(n)}, \ldots, r_{k}^{(n)}$. Define

$$
R_{n}(z):=\left(\frac{1}{n} A_{n} A_{n}^{\mathrm{T}}-z I_{n}\right)^{-1}
$$

and

$$
Y_{k}:=\mathbb{E}_{\leq k} \frac{1}{n} \operatorname{Tr} R_{n}(z)
$$

for $k=0,1, \ldots, n$. Then $\left\{Y_{k}\right\}_{k=0}^{n}$ is a martingale since $\mathbb{E}_{\leq k} Y_{k+1}=Y_{k}$. Define the martingale difference sequence

$$
\alpha_{k}:=Y_{k}-Y_{k-1}
$$


for $k=1,2, \ldots, n$. We then note that

$$
\sum_{k=1}^{n} \alpha_{k}=\frac{1}{n} \operatorname{Tr} R_{n}(z)-\mathbb{E} \frac{1}{n} \operatorname{Tr} R_{n}(z) .
$$

We will bound the $p$-th moment of the sum in (17), but first we obtain a bound on the individual summands $\alpha_{k}$.

For each $1 \leq k \leq n$, define the set

$$
J(k):=\left\{1 \leq j \leq n: k \leq j \leq k+\beta_{n}\right\} .
$$

Now let $A_{n, J(k)}$ be obtained from the matrix $A_{n}$ by removing row $j$ if and only if $j \in J(k)$. Let

$$
R_{n, J(k)}(z)=\left(\frac{1}{n} A_{n, J(k)} A_{n, J(k)}^{\mathrm{T}}-z I_{n}\right)^{-1} .
$$

A simple computation (see for instance [12, Example 5.1.5]) reveals that $\mathbb{E}_{\leq k} \operatorname{Tr} R_{n, J(k)}(z)=$ $\mathbb{E}_{\leq k-1} \operatorname{Tr} R_{n, J(k)}(z)$ by condition (vii) of Definition 1. Thus

$$
\alpha_{k}=\mathbb{E}_{\leq k} \frac{1}{n}\left(\operatorname{Tr} R_{n}(z)-\operatorname{Tr} R_{n, J(k)}(z)\right)-\mathbb{E}_{\leq k-1} \frac{1}{n}\left(\operatorname{Tr} R_{n}(z)-\operatorname{Tr} R_{n, J(k)}(z)\right) .
$$

Using the triangle inequality and equation (3.11) in [7, we have that

$$
\left|\operatorname{Tr} R_{n}(z)-\operatorname{Tr} R_{n, J(k)}(z)\right| \leq \frac{\beta_{n}+1}{|\operatorname{Im} z|}
$$

and hence

$$
\left|\alpha_{k}\right| \leq \frac{2\left(\beta_{n}+1\right)}{n|\operatorname{Im} z|}
$$

We now apply the Burkholder inequality (see for example [8, Lemma 2.12] for a complex-valued version of the Burkholder inequality) and obtain that there exists a constant $C_{p}>0$ such that

$$
\begin{aligned}
\mathbb{E}\left|\sum_{k=1}^{n} \alpha_{k}\right|^{p} & \leq C_{p} \mathbb{E}\left(\sum_{k=1}^{n}\left|\alpha_{k}\right|^{2}\right)^{p / 2} \\
& \leq C_{p}\left(\frac{4\left(\beta_{n}+1\right)^{2} n}{n^{2}|\operatorname{Im} z|^{2}}\right)^{p / 2} \\
& \leq \frac{C_{p} 2^{p}\left(\beta_{n}+1\right)^{p}}{n^{p / 2}|\operatorname{Im} z|^{p}} .
\end{aligned}
$$

Acknowledgment. The author is grateful to A. Litvak for pointing out Theorem 3.13 in [2]. The author would also like to thank A. Soshnikov and D. Renfrew for useful conversations and comments.

\section{REFERENCES}

[1] Adamczak, R., On the Marchenko-Pastur and circular laws for some classes of random matrices with dependent entries, Electronic Journal of Probability, Vol. 16 (2011).

[2] Adamczak, R.; Litvak, A.; Pajor, A.; Tomczak-Jaegermann, N., Quantitative estimates of the convergence of the empirical covariance matrix in log-concave ensembles, J. Amer. Math. Soc. 23 (2010), 535-561.

[3] Anderson G.W., Guionnet A., and Zeitouni O. An Introduction to Random Matrices, Cambridge Studies in Advanced Mathematics 118, Cambridge University Press, New York, 2010. 
[4] Aubrun, G., Random points in the unit ball of $l_{p}^{n}$, Positivity, 10(4):755-759, 2006.

[5] Bai, Z. D. Methodologies in spectral analysis of large-dimensional random matrices, a review. Statist. Sinica 9, 611-677 (1999).

[6] Bai, Z. D., Hu, J., Pan, G., Zhou, W., A Note on Rate of Convergence in Probability to Semicircular Law, Electronic Journal of Probability, Vol. 16(2011).

[7] Z. D. Bai, Convergence Rate of Expected Spectral Distributions of Large Random Matrices. Part I. Wigner Matrices, Ann. Probab. Volume 21, Number 2 (1993), 625-648.

[8] Z. D. Bai, J. Silverstein, Spectral analysis of large dimensional random matrices, Mathematics Monograph Series 2, Science Press, Beijing 2006.

[9] Bordenave, B., Caputo, P., Chafa, D., Circular law theorem for random Markov matrices, Probability Theory and Related Fields, DOI 10.1007/s00440-010-0336-1 (2011).

[10] Chafai, D., The Dirichlet Markov Ensemble, J. Multivariate Anal. 101 (2010), no. 3, 555-567.

[11] Chatterjee, S., Diaconis P., Sly A., Properties of Uniform Doubly Stochastic Matrices, arXiv:1010.6136 1 [math.PR]

[12] Durrett R., Probability. Theory and Examples, 4th ed., Cambridge University Press, New York, 2010.

[13] Götze, F.; Tikhomirov, A.; Limit theorems for spectra of positive random matrices under dependence, Zap. Nauchn. Sem. S.-Petersburg. Otdel. Mat. Inst. Steklov. (POMI), Vol. 311 (2004), Veroyatn. i Stat.7, 92-123, 299.

[14] Götze, F.; Tikhomirov, A.; On the Rate of Convergence to the Marchenko-Pastur Distribution, arXiv:1110.1284 1 [math.PR].

[15] Götze, F.; Tikhomirov, A.; Limit Theorems for spectra of random matrices with martingale structure, Stein's Method and Applications, Singapore Univ. Press (2005), pp. 181-195.

[16] R. Horn, Ch. Johnson, Topics in Matrix analysis, Cambridge University Press, 1991.

[17] V. Marchenko, L. Pastur, Distribution of eigenvalues of some sets of random matrices, Math USSR-Sb. 1, (1967), 457-486.

[18] M.L. Mehta, Random Matrices, 3rd ed., Elsevier/Academic Press, Amsterdam, 2004.

[19] Mendelson, Pajor, On singular values of matrices with independent rows, Bernoulli 12(5), 2006, 761-773.

[20] Pajor, A.,, Pastur, L., On the Limiting Empirical Measure of the sum of rank one matrices with log-concave distribution, arXiv:0710.1346v1 [math.PR].

[21] Wachter, K. W., The strong limits of random matrix spectra for sample matrices of independent elements, Ann. Probab. 6, 1-18 (1978).

[22] Y. Q. Yin, P. R. Krishnaiah, Limit Theorem for the Eigenvalues of the Sample Covariance Matrix when the Underlying Distribution is Isotropic, Theory Probab. Appl. 30, pp. 861-867.

Department of Mathematics, Rutgers, Piscataway, NJ 08854

E-mail address: sdo21@math.rutgers.edu 